
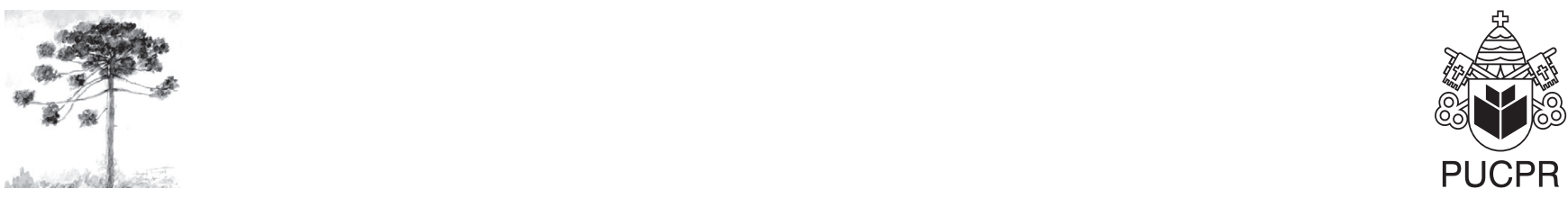

PUCPR

\title{
Variação da avifauna, em relação ao nível da maré, no uso de um plano intermareal no Mar Pequeno, Ilha Comprida, São Paulo, Brasil
}

\author{
Bird variation, in relation to the level of the tide, in the use of a plan intertidal \\ at Mar Pequeno, Ilha Comprida, São Paulo, Brazil
}

\author{
Gimel Roberto Zanin ${ }^{[a]}$, Luciana Finotti Tosin ${ }^{[\mathrm{b}]}$, Edison Barbieri ${ }^{[\mathrm{c}]}$
}

[a] Bacharel em Oceanografia pelo Instituto Oceanográfico da Universidade de São Paulo, São Paulo, SP - Brasil, e-mail: gimel_broz@yahoo.com.br

[b] Bacharel em Ciências Biológicas pelo Instituto de Biociências da Universidade de São Paulo (USP), São Paulo, SP - Brasil, e-mail: luftos@yahoo.com

[c] Doutorado em Oceanografia (Oceanografia Biológica) pela Universidade de São Paulo (USP), Professor e Pesquisador do Instituto de Pesca, Cananeia, SP - Brasil, e-mail: edisonbarbieri@yahoo.com.br

\section{Resumo}

O estuário é um importante local de alimentação e repouso para bandos mistos de aves. Por esse motivo, observações diárias foram feitas no Baixio do Boguaçu, na Ilha Comprida, em duas campanhas durante a maré de quadratura e de sizígia, no mês de janeiro de 2007. Os resultados mostram a presença, dentre 2.151 aves observadas, de 14 espécies, das quais 46,95\% pertenceram à espécie Phalacrocorax brasilianus, 20,41\% à Egretta caerulea e 17,01\% à Fregata magnificens. O índice de diversidade de Shannon variou entre 1,6128 e 0,8421, enquanto o índice de equitabilidade de Pielou oscilou entre 0,649 e 0,523. Comparadas às variações na amplitude da maré, a riqueza e a abundância total de espécies apresentaram correlações negativas de 0,557 e 0,4281, respectivamente, no Baixio do Boguaçu. As espécies que apresentaram maiores valores de correlação com as variações no nível médio do mar foram: Egretta caerulea $(0,6955)$, Platalea ajaja (0,646), Ardea cocoi (0,4756), Larus dominicanus (0,3033), Ardea alba (0,2245), Calidris fuscicollis $(0,2101)$ e Eudocimus ruber $(0,1786)$.

Palavras-chave: Avifauna. Estuário. Mangue. Ilha Comprida. 


\begin{abstract}
The estuary is an important place of feeding and rest for mixing flocks of waterbirds. Daily record had been made in the sand bank, in two campaigns during the sizigia and quadrature tide, in January of 2007. The results show the presence, amongst 2.151 observed birds, of 14 species, which $46.95 \%$ of the birds had belonged to the Phalacrocorax brasilianus, 20.41\% to Egretta caerulea and 17, 01\% to Fregata magnificens. The index of diversity of Shannon varied between 1,6128 and 0,8421, while the index of equitability of Pielou oscillated between 0,649 and 0,523. Compared with the variations in the amplitude of the tide, the wealth and the total abundance of species they had presented negative correlations of 0,557 and 0,4281, respectively, in the Sand bank of the Boguaçu. The species that had presented greaters values of correlation with the variations in the mean sea level had been: Egretta caerulea $(0,6955)$, Platalea ajaja $(0,646)$, Ardea cocoi $(0,4756)$, Larus dominicanus $(0,3033)$, Ardea alba $(0,2245)$, Calidris fuscicollis $(0,2101)$ and Eudocimus ruber $(0,1786)$.
\end{abstract}

Keywords: Birds. Estuary. Mangrove. Ilha Comprida.

\section{Introduçáo}

O Complexo Estuarino Lagunar de CananeiaIguape-Ilha Comprida está localizado no extremo sul do Estado de São Paulo e apresenta grande complexidade ambiental, envolvendo uma intrincada rede de interações bióticas e abióticas que condicionam altos níveis de produtividade (1). Ao longo de toda a sua extensão, recebe água doce de numerosos e diversos rios, sendo mais expressivo o Rio Ribeira de Iguape.

Os estuários constituem corpos de água semifechados, com conexão livre para o oceano aberto, onde podem ocorrer desembocaduras fluviais, o que faz com que suas águas sofram diluição significativa de salinidade (2). Essa mistura de águas doce e salgada cria o ambiente propício para a existência de ecossistemas costeiros de transição entre o meio terrestre e marinho, os manguezais (3).

O Complexo Estuarino Lagunar de Cananeia-Iguape-Ilha Comprida, que inclui a área de reserva natural formada pela Mata Atlântica, as ilhas de Cananeia, Comprida e do Cardoso, além do manguezal, representa um dos mais importantes ecossistemas costeiros brasileiros (3), que necessita ser preservado. Por isso, apesar do impacto antrópico causado pelo afluxo de efluentes domésticos e a pesca, esse complexo abriga uma grande diversidade de aves.

O estuário de Cananeia-Iguape-Ilha Comprida atua como um importante local de alimentação e repouso para bandos mistos de aves marinhas, limícolas e habitantes de borda (4), e os baixios encontrados nessa região facilitam a alimentação de algumas dessas aves durante a maré baixa.
Em virtude da carência de informações sobre a utilização desses baixios pela avifauna, esse trabalho teve como objetivo descrever a abundância específica de aves marinhas no Baixio do Boguaçu, na Ilha Comprida, caracterizando a variação diária da estrutura da comunidade em relação à variação do nível da maré.

\section{Materiais e métodos}

\section{Descrição da área}

O Baixio do Boguaçu é uma planície intermareal de sedimentos lodosos envolta pelo mangue - que possui vegetação característica e fauna com adaptações exclusivas para a variação de salinidade e a duração do período de inundação (5). À medida que essa salinidade diminui e/ou as águas se tornam muito turbulentas, o mangue vai gradativamente desaparecendo (6).

Nesse ambiente, tipicamente salobro, do sul de São Paulo, três espécies garantem, segundo a sequência, a característica de vegetação pioneira: Rhizophora mangle, Avicennia schaueriana e Laguncularia racemosa. Essas espécies ocorrem em toda a região lagunar, destacando a maior presença de $R$. mangle na primeira porção do mangue. Normalmente, a $L$. racemosa aparece só nos terrenos mais firmes, em áreas mais resguardadas da maré, nas planícies salobras e nos rios com drenagem dificultada (6). Contudo, de forma geral, o manguezal apresenta composição florística homogênea ao longo de todo o litoral (7). 


\section{Amostragem}

$\mathrm{Na}$ área de estudo foram realizadas duas campanhas, com intervalo de uma semana, compreendendo dois dias de amostragem cada, durante o mês de janeiro de 2007. Em cada dia, foram feitas 8 observações, com 10 minutos cada, intercaladas com intervalos de 50 minutos.

Para o levantamento quantitativo foi utilizado o método do ponto fixo (8). Nessa amostragem por pontos, foram consideradas todas as detecções com uma distância ilimitada, anotando-se todas as espécies vistas, independente da distância. No caso de limite do ambiente estudado, foram registradas somente as espécies incluídas no sítio amostrado. Como informação adicional das espécies registradas, anotou-se o comportamento da ave no momento da observação (forrageamento, voo, repouso, alimentação). Os dados quantitativos expressam a frequência de uso de uma espécie em um determinado ambiente. Para o registro dos contatos visuais, foi utilizado um binóculo $8 \times 30$.

A riqueza específica foi obtida a partir de estudos quantitativos e observações sistemáticas no sítio de estudo. A nomenclatura das espécies seguiu Sick (9). Além dos dados biológicos, foram obtidos, para cada dia de amostragem, os registros do nível do mar na região em estudo, a fim de relacionar as variações específicas das aves marinhas com as oscilações diárias da maré. Os dados foram cedidos pelo Instituto Oceanográfico da USP.

\section{Análise dos dados}

Uma vez que o protocolo de coleta dos dados foi igual para todas as amostragens realizadas, o número de espécies foi utilizado como medida de riqueza de espécies e o número de indivíduos registrados, como índice de abundância relativa. O índice de diversidade de Shannon (10),

$$
\mathrm{H}^{\prime}=-\sum \mathrm{pi} \ln \mathrm{pi}
$$

sendo pi $=$ ni $/ \mathrm{N}$ a proporção de indivíduos encontrados na espécie i, e o índice de equitabilidade de Pielou foram calculados como informação adicional:

$$
e=\frac{\mathrm{H}^{\prime}}{\ln \mathrm{S}}
$$

em que S é o número de espécies.

Para os dados de maré foram considerados os valores do nível do mar durante o intervalo, nos quais houve observação da avifauna.

Foi feita uma análise de correlação de Spearman (11) a fim de determinar a influência das variações diárias no nível do mar sobre a riqueza e a abundância total e específica das aves. Os valores de variação do nível do mar foram normalizados pelo fator $\log (\mathrm{x})$, enquanto os valores de abundância de aves, em razão do grande número de valores nulos, foram normalizados pelo fator $\log (\mathrm{x}+1)$.

\section{Resultados}

\section{Avifauna}

Em um total de 32 amostragens, realizadas em quatro dias de observação, 2.151 aves foram observadas, pertencentes a 8 famílias e 13 gêneros. Foram identificadas 14 espécies: Sula leucogaster, Phalacrocorax brasilianus, Fregata magnificens, Ardea alba, Ardea cocoi, Egretta thula, Egretta caerulea, Butorides striatus, Platalea ajaja, Eudocimus ruber, Calidris fuscicollis, Larus dominicanus, Sterna eurygnatha e Ceryle torquata, tendo as espécies $S$. eurygnatha e B. striatus ocorrido somente na primeira campanha, e $C$. fuscicollis e $C$. torquata, somente na segunda.

As Tabelas $1 \mathrm{a}$ e $1 \mathrm{~b}$ apresentam os dados referentes ao número de indivíduos observados para cada espécie, em cada dia de observação, e sua porcentagem relativa, durante a primeira e a segunda campanhas, respectivamente.

Do total observado, $46,95 \%$ das aves pertenceram à espécie P. brasilianus, 20,41\% à E. caerulea, $17,01 \%$ à F. magnificens e 5,67\% à $A$. alba, 2,60\% à $C$. fuscicollis, 2,23\% à E. thula, 1,81\% à $P$. ajaja e 1,67\% à $A$. cocoi. As demais espécies apresentaram porcentagens inferiores a $1 \%$.

$\mathrm{Na}$ primeira campanha ( $\mathrm{n}=1.065), 12$ espécies foram identificadas, sendo $49,25 \%$ das aves pertencentes à $P$. brasilianus, $23,47 \%$ à $F$. magnificens e $14,65 \%$ à E. caerulea. No primeiro dia de observação, 12 espécies foram identificadas, com predomínio de P. brasilianus (42,14\%), seguida de E. caerulea $(27,86 \%)$. No segundo dia, 5 espécies, com grande dominância de P. brasilianus (57,03\%) e F. magnificens (39,41\%), e ausência de E. caerulea (Figura 1). 
Tabela 1a - Número de indivíduos, e respectiva porcentagem, para cada espécie identificada no Baixio do Boguaçu na primeira campanha

\begin{tabular}{|c|c|c|c|c|c|c|}
\hline \multirow[b]{2}{*}{ Família } & \multirow[b]{2}{*}{ Espécie } & & \multicolumn{2}{|c|}{$1^{\circ}$ dia } & \multicolumn{2}{|c|}{$2^{\circ}$ dia } \\
\hline & & & $\mathbf{N}$ & $\%$ & $\mathbf{N}$ & $\%$ \\
\hline Sulidae & Sula lencogaster & (Boddaert, 1783) & 2 & 0,36 & - & - \\
\hline Phalacrocoracidae & Phalacrocorax brasilianus & (Gmelin, 1789) & 236 & 42,14 & 288 & 57,03 \\
\hline Fregatidae & Fregata magnificens & Mathews, 1914 & 51 & 9,11 & 199 & 39,41 \\
\hline \multirow{5}{*}{ Ardeidae } & Ardea alba & (Pallas, 1764) & 52 & 9,29 & 9 & 1,78 \\
\hline & Ardea cocoi & Linnaeus, 1766 & 14 & 2,50 & - & - \\
\hline & Egretta thula & (Molina, 1782) & 13 & 2,32 & 3 & 0,59 \\
\hline & Florida caerulea & (Linnaeus, 1758) & 156 & 27,86 & - & - \\
\hline & Butorides striatus & (Linnaeus, 1758) & 1 & 0,18 & - & - \\
\hline \multirow[t]{2}{*}{ Threskiornithidae } & Platalea ajaja & Linnaeus, 1758 & 21 & 3,75 & 6 & 1,19 \\
\hline & Eudocimus ruber & (Linnaeus, 1758) & 4 & 0,71 & - & - \\
\hline Scolopacidae & Calidris fuscicollis & (Vieillot, 1819) & - & - & - & - \\
\hline \multirow[t]{2}{*}{ Laridae } & Larus dominicanus & Lichtenstein, 1823 & 5 & 0,89 & - & - \\
\hline & Sterna eurygnatha & Saunders, 1876 & 5 & 0,89 & - & - \\
\hline Alcendinidae & Ceryle torquata & (Linnaeus, 1766) & - & - & - & - \\
\hline Total & & & 560 & 100,00 & 505 & 100,00 \\
\hline
\end{tabular}

Fonte: Dados da pesquisa.

Tabela 1b - Número de indivíduos, e respectiva porcentagem, para cada espécie identificada no Baixio do Boguaçu na segunda campanha

\begin{tabular}{|c|c|c|c|c|c|c|}
\hline \multirow[b]{2}{*}{ Família } & \multirow[b]{2}{*}{ Espécie } & & \multicolumn{2}{|c|}{$1^{\circ}$ dia } & \multicolumn{2}{|c|}{$2^{\circ}$ dia } \\
\hline & & & $\mathbf{N}$ & $\%$ & $\mathbf{N}$ & $\%$ \\
\hline Sulidae & Sula leucogaster & (Boddaert, 1783) & 2 & 0,29 & 4 & 1,01 \\
\hline Phalacrocoracidae & Phalacrocorax brasilianus & (Gmelin, 1789) & 261 & 37,77 & 225 & 56,96 \\
\hline Fregatidae & Fregata magnificens & Mathews, 1914 & 22 & 3,18 & 94 & 23,80 \\
\hline \multirow[t]{5}{*}{ Ardeidae } & Ardea alba & (Pallas, 1764) & 29 & 4,20 & 32 & 8,10 \\
\hline & Ardea cocoi & Linnaeus, 1766 & 14 & 2,03 & 8 & 2,03 \\
\hline & Egretta thula & (Molina, 1782) & 16 & 2,32 & 16 & 4,05 \\
\hline & Florida caerulea & (Linnaeus, 1758) & 268 & 38,78 & 15 & 3,80 \\
\hline & Butorides striatus & (Linnaeus, 1758) & - & - & - & - \\
\hline \multirow[t]{2}{*}{ Threskiornithidae } & Platalea ajaja & Linnaeus, 1758 & 11 & 1,59 & 1 & 0,25 \\
\hline & Eudocimus ruber & (Linnaeus, 1758) & 3 & 0,43 & - & - \\
\hline Scolopacidae & Calidris fuscicollis & (Vieillot, 1819) & 56 & 8,10 & - & - \\
\hline \multirow[t]{2}{*}{ Laridae } & Larus dominicanus & Lichtenstein, 1823 & 7 & 1,01 & - & - \\
\hline & Sterna eurygnatha & Saunders, 1876 & - & - & - & - \\
\hline Alcendinidae & Ceryle torquata & (Linnaeus, 1766) & 2 & 0,29 & - & - \\
\hline Total & & & 691 & 100,00 & 395 & 100,00 \\
\hline
\end{tabular}

Fonte: Dados da pesquisa. 


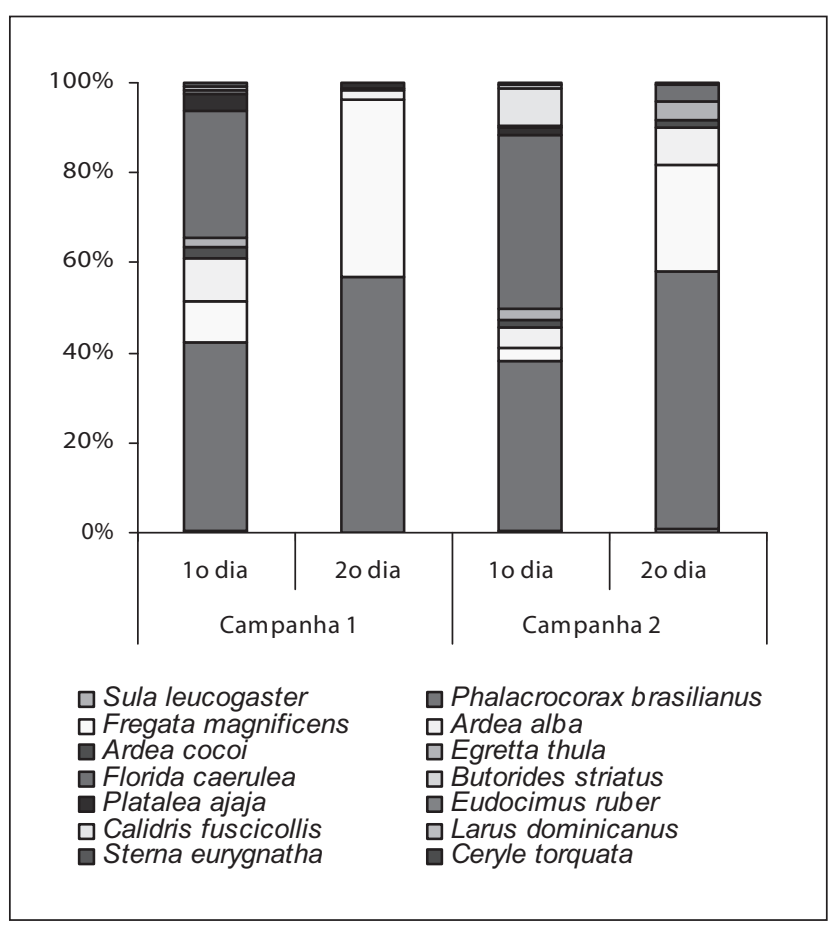

Figura 1 - Abundância relativa de cada espécie identificada, em cada dia de coleta, no Baixio do Boguaçu

Fonte: Dados da pesquisa.
$\mathrm{Na}$ segunda campanha ( $\mathrm{n}=1.086), 12$ espécies foram identificadas, sendo $44,75 \%$ das aves pertencentes à $P$. brasilianus, 26,06\% à E. caerulea e 10,68\% à F. magnificens. No primeiro dia de observação, 12 espécies foram identificadas, com predomínio de E. caerulea $(38,78 \%)$, seguida de P. brasilianus $(37,77 \%)$. No segundo dia, 8 espécies, com grande dominância de P. brasilianus $(56,96 \%)$ e F. magnificens $(23,80 \%)$, seguida de $A$. alba $(8,10 \%)$ (Figura 1$)$.

São apresentados os dados de abundância relativa das espécies identificadas nos dois dias de observação, durante a primeira (Figura 2) e a segunda (Figura 3) campanhas, para cada amostragem realizada. É possível notar que, no primeiro dia de observação, tanto na primeira quanto na segunda campanha a porcentagem relativa de P. brasilianus no total de aves observadas aumenta com o passar do dia, e a porcentagem relativa de E. caerulea apresenta um máximo entre 10 e 11 horas, diminuindo com o passar do dia. Já no segundo dia, principalmente na primeira campanha, há menor variação nas porcentagens relativas das espécies, sendo a dominância de $P$. brasilianus e F. magnificens mantida durante o período de amostragem, com poucas exceções (Figuras 2 e 3).

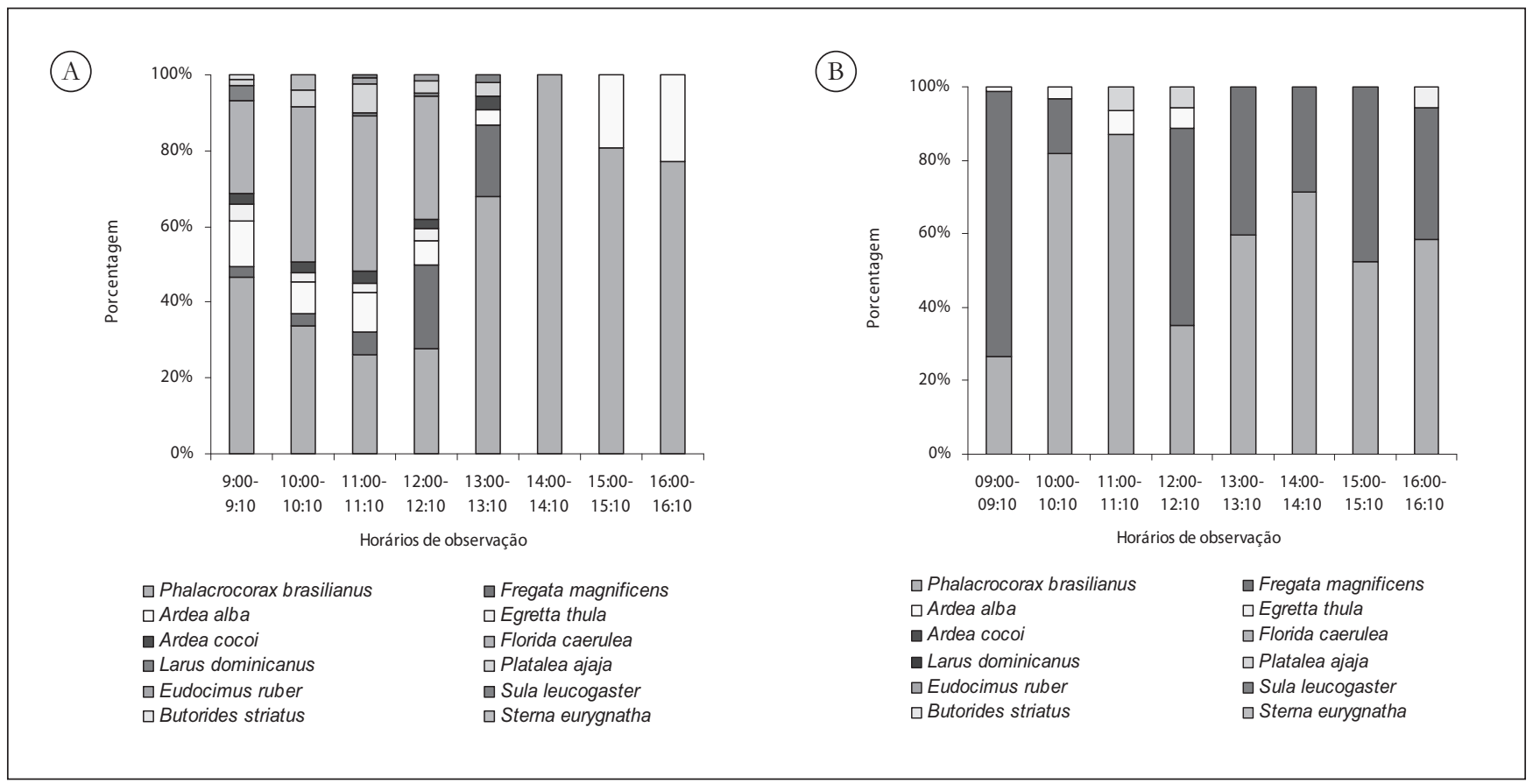

Figura 2 - Abundância relativa das aves identificadas no primeiro (a) e no segundo (b) dias de observação, em cada período amostrado na primeira campanha. no Baixio do Boguaçu

Fonte: Dados da pesquisa. 


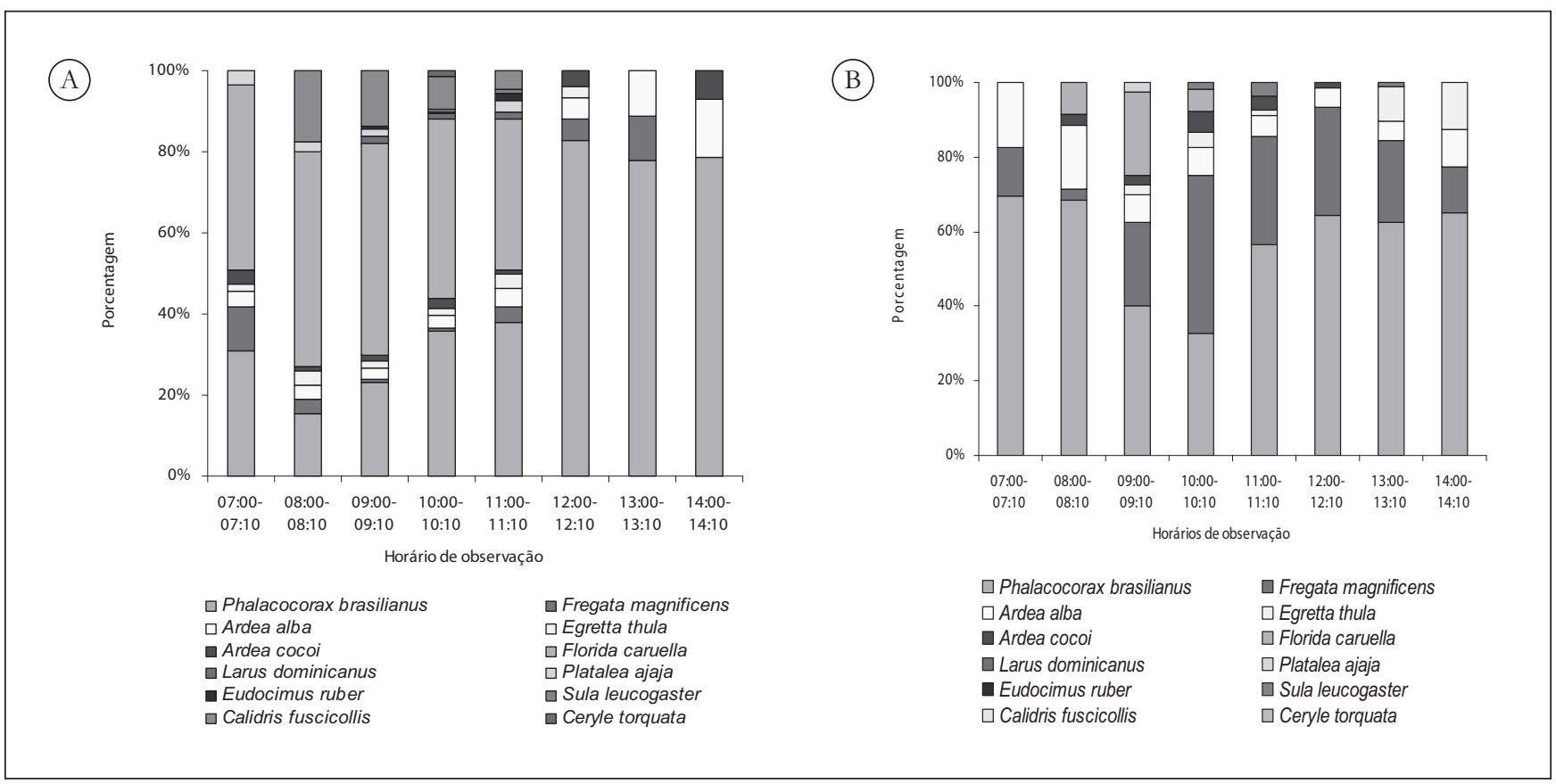

Figura 3 - Abundância relativa das aves identificadas no primeiro (a) e no segundo (b) dias de observação, em cada período amostrado na segunda campanha, no Baixio do Boguaçu

Fonte: Dados da pesquisa.

A Tabela 2 apresenta os comportamentos observados para cada espécie durante os períodos de observação, considerando as duas campanhas realizadas. Nota-se que a espécie $P$. brasilianus apresentou diversos comportamentos, estando a maior parte dos indivíduos, inclusive nos períodos nos quais representou a maior abundância relativa dentre as espécies, associada a fragmentos de madeira emersos provenientes de antigos cercos ou a cercos em atividade. A espécie F. magnificens foi observada, em todo o período amostrado, sobrevoando a área estudada; já as espécies $A$. alba, P. ajaja e $A$. cocoi, de maior porte e com pernas mais alongadas, foram observadas durante todo o período, com abundâncias relativas baixas, geralmente em repouso ou se alimentando no baixio; e as espécies E. caerulea e C. fuscicollis, de menor porte e com pernas curtas, foram observadas somente em um período do dia, em repouso ou alimentando-se.

\section{Dados hidrográficos}

As Figuras 4 e 5 apresentam as variações no nível do mar e da riqueza de espécies durante o período de amostragem, para cada dia observado. A amplitude de maré na área de estudo é de cerca de 1,20 m. Os menores valores da altura de maré foram registrados no primeiro dia de amostragem, tanto na primeira quanto na segunda campanha, enquanto os valores mais elevados ocorreram no segundo dia de cada campanha. Quando o nível do mar alcança um nível suficientemente baixo para as espécies de aves se alimentarem no substrato, toda a área superior do baixio torna-se disponível para repouso e alimentação, como ocorreu no primeiro dia de amostragem em cada campanha. Quando o nível do mar sobe e atinge um nível superior à profundidade de forrageamento para a maioria das espécies, o baixio passa a se tornar não disponível.

\section{Análise dos dados}

Os índices de diversidade de Shannon e de equitabilidade de Pielou foram calculados para cada dia de coleta. Para o índice de Shannon, no primeiro e no segundo dia de coleta, foram obtidos os valores 1,6128 e 0,8421, na primeira campanha, e 1,5176 e 1,2605, na segunda, respectivamente. 
Quanto ao índice de Pielou, os valores encontrados foram: 0,649 para o primeiro dia e 0,523 para o segundo, durante a primeira campanha; e 0,610 para o primeiro dia e 0,606 para o segundo, na segunda campanha.

A riqueza, a abundância total e a abundância relativa de aves, em cada amostragem, foram comparadas com os dados de variação no nível médio do mar por meio de regressão simples. Considerando todas as amostragens realizadas, a análise indicou, comparando-se às oscilações do nível do mar, uma correlação negativa de 0,557 para a riqueza e uma correlação negativa de 0,4281 para a abundância total de aves no Baixio do Boguaçu. As espécies que apresentaram maiores valores de correlação com as variações no nível médio do mar foram: $E$. caerulea (0,6955), P. ajaja (0,646), A. cocoi $(0,4756)$, $L$. dominicanus $(0,3033), A$. alba $(0,2245)$, C. fuscicollis $(0,2101)$ e E. ruber $(0,1786)$. As demais espécies não apresentaram valores significativos.

Tabela 2 - Comportamento das aves identificadas no Baixio do Boguaçu durante a primeira e a segunda campanhas (em porcentagem)

\begin{tabular}{lcccc}
\hline Espécie & $\begin{array}{c}\text { Fragmentos } \\
\text { emersos de madeira }\end{array}$ & $\begin{array}{c}\text { Alimentação/ } \\
\text { repouso na água }\end{array}$ & $\begin{array}{c}\text { Alimentação/ } \\
\text { repouso no baixio }\end{array}$ & Vôo \\
\hline Sula lencogaster & - & - & 45,46 & 54,54 \\
Phalacrocorax brasilianus & 91,98 & 4,59 & 2,36 & 1,07 \\
Fregata magnificens & - & - & 0,14 & 99,86 \\
Ardea alba & 11,90 & - & 85,72 & 2,38 \\
Ardea cocoi & - & - & 66,67 & 33,33 \\
Egretta thula & 25,80 & - & 74,20 & - \\
Florida caerulea & - & - & 99,51 & 0,49 \\
Butorides striatus & - & - & 100,00 & - \\
Platalea ajaja & - & - & 76,92 & 0,23 \\
Eudocimus ruber & - & - & 71,43 & 28,57 \\
Calidris fuscicollis & - & - & 100,00 & - \\
Larus dominicanus & - & - & 80,00 & 20,00 \\
Sterna eurygnatha & - & - & - & 100,00 \\
Ceryle torquata & - & - & 100,00 & - \\
\hline
\end{tabular}

Fonte: Dados da pesquisa.
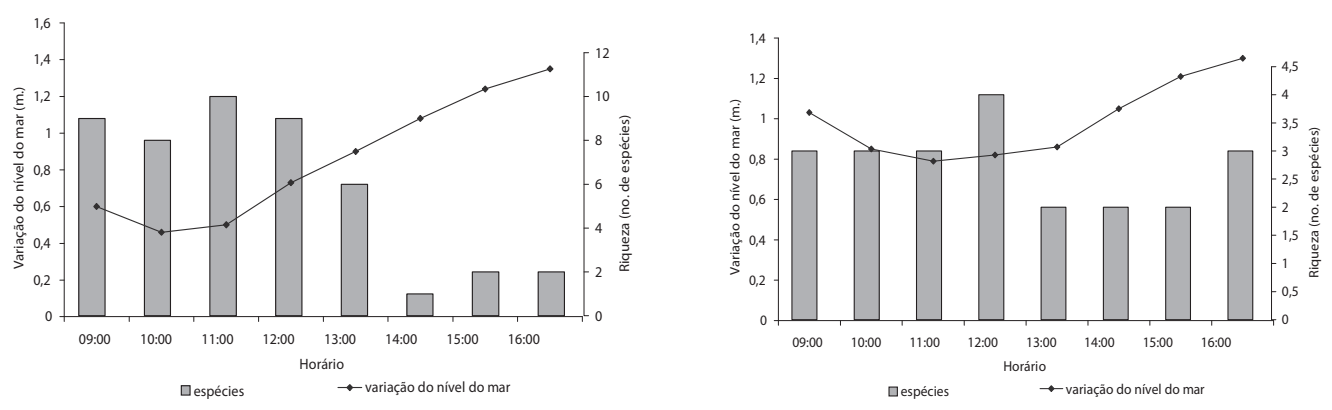

Figura 4 - Dados das variações da altura do mar durante o primeiro e o segundo dia de observação, na primeira campanha no Baixio do Boguaçu

Fonte: Dados da pesquisa. 


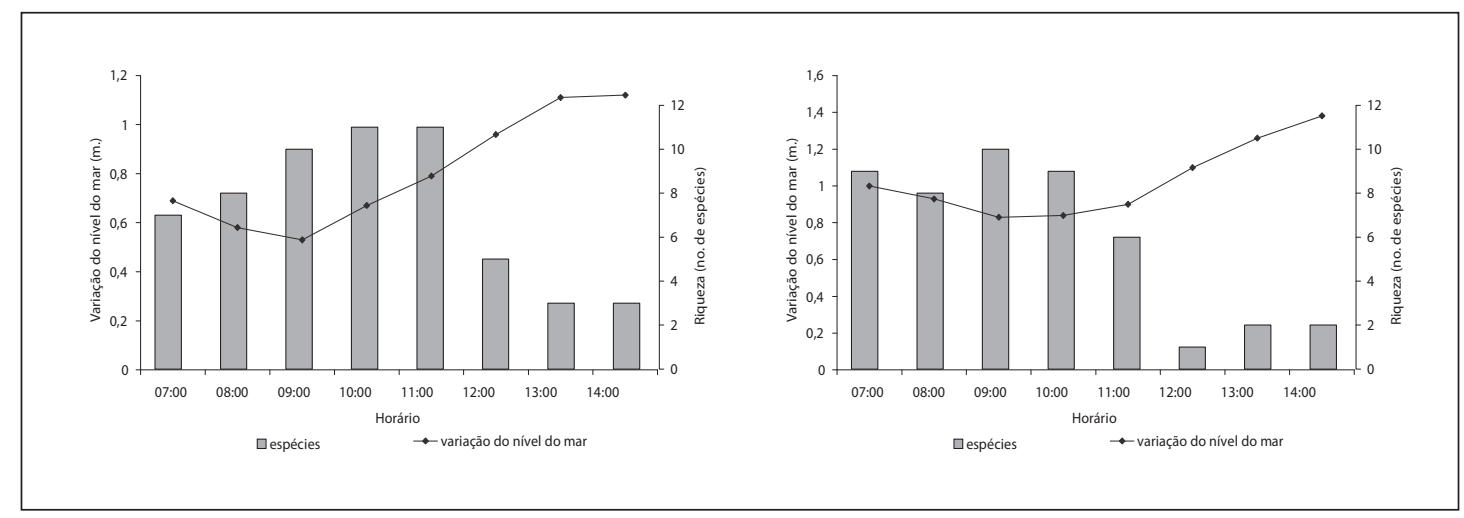

Figura 5 - Dados das variações da altura do mar durante o primeiro e segundo dia de observação, na segunda campanha no Baixio do Boguaçu

Fonte: Dados da pesquisa.

\section{Discussáo}

Em ambientes de influência marítima, agrupamentos heteroespecíficos de aves, em geral, são formados em decorrência da partilha de alimento, local adequado para repouso e nidificação, o que contribui na proteção do grupo. As aves costeiras são consideradas espécies visitantes e importantes predadoras de topo do intermareal de fundos moles (12). O complexo estuarino, no qual se insere o baixio amostrado, recebeágua do mare dos rios provenientes do interior do continente e que ali deságuam. Esse ambiente é caracterizado pela formação de manguezal, caracterizada pela presença dominante de três espécies vegetais: $R$. mangle, $A$. shaneriannae $L$. racemosa (13). Tratando-se de um ambiente rico em matéria orgânica e em fauna marinha, representa significativa fonte alimentar para aves, como P. brasilianus, Freagata magnificens, C. fuscicollis, todos os Ardeidae registrados, que permanecem em seus limites durante algumas horas do dia pescando e/ou repousando. Moraes e Krul (12), estudando a distribuição de aves no litoral do Paraná, observaram que as espécies B. striatus e $C$. torquata estiveram restritas a esse tipo de ambiente.

As aves limícolas pertencentes às famílias Phalacrocoracidae e Ardeidae foram responsáveis pelas maiores abundâncias ao longo deste estudo. Um resultado semelhante foi encontrado por Branco (13), estudando o estuário do Saco da Fazenda, em Santa Catarina. Olmos (14), trabalhando com a avifauna da baixada do polo industrial de Cubatão, São Paulo, constatou que a metade das espécies observadas apresentou hábitos palustres.
O índice de diversidade de Shannon, largamente utilizado nos estudos de comunidades, parece não ser frequentemente utilizado nos trabalhos de avifauna de locais alagados (15). Apesar disso, os valores diários no Baixio do Boguaçu variaram entre 0,84 e 1,61. Em seu trabalho, Branco (13) encontrou valores mensais oscilando entre 0,36 e 2,31.

A grande representatividade da espécie $P$. brasilianus se deve, principalmente, à presença dos fragmentos emersos de madeira oriundos de atividades de pesca com cercos-fixos na região em estudo. Poucos indivíduos foram observados em atividade de voo ou alimentação no baixio. Seus ninhos são construídos sobre árvores, nas proximidades de ambientes aquáticos (9), e existem poucas informações disponíveis sobre sua biologia e abundância no País.

A espécie E. caerulea esteve sempre relacionada com períodos de maré baixa, apresentando o maior índice de correlação com a altura da maré. Como mostrado, as aves dessa espécie utilizam o baixio para alimentação durante os períodos de maré baixa, desaparecendo gradativamente, com o aumento do nível do mar, graças à progressiva indisponibilidade de áreas de alimentação e repouso no baixio.

As espécies $A$. cocoi e $A$. alba estiveram associadas a todos os níveis de maré, apresentando abundância mais elevada nos períodos de maré baixa e ocorrendo em menores quantidades durante as maiores alturas de maré. Essas espécies possuem pernas e bicos mais alongados que as demais e permanecem no baixio se alimentando por um intervalo de tempo maior. Por isso, as populações dessas aves se distribuem por um grande intervalo 
de níveis de maré, não apresentando concentração num determinado nível.

A C. fuscicollis foi observada somente na segunda campanha, sendo representada no primeiro dia de coleta, em um estreito intervalo de alturas de maré. Na Figura 2, é possível observar que ocorre diminuição gradativa na participação dessa espécie na composição de aves no baixio com o aumento do nível do mar. Como se tratam de aves de pequeno porte, com pés e bicos curtos, pequenas variações na altura do nível do mar já tornam o baixio indisponível a elas.

A espécie F. magnificens, por sua vez, foi observada sempre em atividade de voo sobre o baixio. Durante os períodos de maré baixa, principalmente no primeiro dia da primeira campanha, essa espécie foi observada alimentando-se no entorno do baixio. Nesse mesmo período, ainda, um grupo de botos foi observado no entorno do baixio e, junto deles, as espécies F. magnificens (3), L. dominicanus (1), P. brasilianus (3) e C. torquata (1) foram observadas em atividade de pesca. Nos demais dias, a F. magnificens não foi observada pescando.

Durante todo o período amostrado, um grande número de embarcações pequenas foi observado navegando próximo ao baixio, o que fez com que algumas espécies se assustassem e levantassem voo. Na manhã do primeiro dia amostrado, foi observado o maior número de espécies no baixio, fato coincidente com os níveis mais baixos de maré e com a ausência de embarcações nas proximidades do baixio.

\section{Conclusóes}

O Baixio do Boguaçu foi visitado, durante o período amostrado, por um grande número de espécies e indivíduos, o que atesta sua importância como local de alimentação e repouso para aves.

A ocorrência e a abundância de uma espécie estão relacionadas com o grau de disponibilidade do baixio para ela, sendo essa característica particular para cada espécie. A variação diária da composição da população no Baixio do Boguaçu está intimamente relacionada com as variações do nível do mar. Há a necessidade de mais estudos quanto à composição da avifauna nos diversos ambientes costeiros e dos fatores ambientais que condicionam a sua distribuição.

O monitoramento dos agrupamentos, nos próximos anos, poderá contribuir para a compreen- são das interações entre as espécies e para a avaliação da importância das populações do estuário, no contexto regional.

\section{Referências}

1. Secretaria do Meio Ambiente - SMA. Planos de manejo nas unidades de conservação, Parque Estadual da Ilha do Cardoso, fase 1 - plano de gestão ambiental. São Paulo: Secretaria do Meio Ambiente; 1998.

2. Rodrigues MG. Análises dos status de conservação das unidades de paisagem no Complexo EstuarinoLagunar Cananéia-Iguape-Guaraqueçaba. [tese] São Paulo: Universidade de São Paulo, Instituto de Biociências; 1998.

3. Schaeffer-Novelli Y. Manguezais brasileiros. [tese] São Paulo: Instituto Oceanográfico; 1991.

4. Barbieri E, Pinna FV. Distribuição do Trinta-ReisReal (Thalasseus maximus) durante 2005 no estuário de Cananéia-Iguape-Ilha Comprida. Ornitologia Neotropical. 2007;18(2):99-110.

5. Adaime RR. Estrutura, produção e transporte em um manguezal. Simpósio sobre Ecossistema da Costa Sul e Sudeste Brasileira: síntese dos conhecimentos. Cananeia, BSP. Academia de Ciências do Estado de São Paulo. 1987;2(1):80-93.

6. Schaeffer-Novelli Y. Manguezais brasileiros: região Sudeste-Sul. Simpósio sobre Ecossistema da Costa Sul e Sudeste Brasileira: síntese dos conhecimentos. Cananeia, BSP. Academia de Ciências do Estado de São Paulo. 1987;2(1):78.

7. Blondel J, Ferry C, Prochot B. La métode des indices ponctuales d'abondance (I.P.A.) ou des releves d'avifaune par "stations d'écoute". Alauda. Montpelier. 1970;38:55-71.

8. Sick H. Ornitologia brasileira. Rio de Janeiro: Nova Fronteira; 1997.

9. Magurran AE. Ecological diversity and its measurement. New Jersey: Princeton University Press; 1988.

10. Siegel S. Nonparametric statistic for the behavioral sciences. New York: McGraw-Hill; 1956.

11. Knox GA. The ecology of seashores. Boca Raton: CRC Press; 2001. 
12. Moraes VS, Krul R. Aves associadas a ambientes de influência marítima no litoral do Paraná. Arquivos de Biologia e Tecnologia. 1995;38(1):121-34.

13. Branco JO. Avifauna associada ao estuário do Saco da Fazenda, Itajaí, Santa Catarina. Revista Brasileira de Zoologia. 2000;17(2):387-94.

14. Olmos F. A avifauna da baixada do pólo industrial de Cubatão. Revista Brasileira de Biologia. 1989;49(2):373-9.
15. Barbieri E, Paes ET. The birds at Ilha Comprida beach (São Paulo state, Brazil): a multivariate approach. Biota Neotropical. 2008;8(3) [acesso 25 maio 2008]. Disponível em: http://www.biotaneotropica. org.br/v8n3/en/abstract?article+bn00408032008

Recebido: $14 / 10 / 2008$ Received: 10/14/2008

Aprovado: $12 / 10 / 2009$ Approved: 10/12/2009 